\title{
The extreme-ultraviolet stellar characterization for atmospheric physics and evolution (ESCAPE) mission concept
}

France, Kevin, Fleming, Brian, Drake, Jeremy, Mason, James, Youngblood, Allison, et al.

Kevin France, Brian T. Fleming, Jeremy J. Drake, James P. Mason, Allison Youngblood, Vincent Bourrier, Luca Fossati, Cynthia S. Froning, Tommi Koskinen, Nicholas Kruczek, Sarah Lipscy, Randall McEntaffer, Suzanne Romaine, Oswald H. W. Siegmund, Erik Wilkinson, "The extreme-ultraviolet stellar characterization for atmospheric physics and evolution (ESCAPE) mission concept," Proc. SPIE 11118, UV, X-Ray, and Gamma-Ray Space Instrumentation for Astronomy XXI, 1111808 (9 September 2019); doi: $10.1117 / 12.2526859$

Event: SPIE Optical Engineering + Applications, 2019, San Diego, California, United States 


\title{
The Extreme-ultraviolet Stellar Characterization for Atmospheric Physics and Evolution (ESCAPE) mission concept
}

Kevin France*a, Brian T. Fleming ${ }^{\mathrm{a}}$, Jeremy J. Drake ${ }^{\mathrm{b}}$, James P. Mason ${ }^{\mathrm{c}}$, Allison Youngblood ${ }^{\mathrm{c}}$, Vincent Bourrier ${ }^{\mathrm{d}}$, Luca Fossati ${ }^{\mathrm{e}}$, Cynthia S. Froning ${ }^{\mathrm{f}}$, Tommi Koskinen ${ }^{\mathrm{g}}$, Nicholas Kruczek ${ }^{\mathrm{a}}$, Sarah Lipscy $^{\mathrm{h}}$, Randall McEntaffer ${ }^{\mathrm{i}}$, Drew Miles ${ }^{\mathrm{i}}$, Suzanne Romaine ${ }^{\mathrm{b}}$, Oswald H. W. Siegmund ${ }^{\mathrm{j}}$, Erik Wilkinson $^{\mathrm{g}}$

aLaboratory for Atmospheric and Space Physics, University of Colorado, UCB 600, Boulder, CO, USA 80309, *kevin.france@ colorado.edu;

${ }^{\mathrm{b}}$ Smithsonian Astrophysical Observatory, 60 Garden Street, Cambridge MA02138, USA;

'NASA Goddard Space Flight Center, Greenbelt, MD, USA;

dObservatoire de l'Université de Genève, 51 chemin des Maillettes, 1290 Sauverny, Switzerland; 'Space Research Institute, Austrian Academy of Sciences, Schmiedlstrasse 6, A-8042 Graz, Austria; ${ }^{\mathrm{f}}$ McDonald Observatory, University of Texas at Austin, Austin, TX 78712, USA; 'Lunar and Planetary Laboratory, University of Arizona, Tucson, AZ, USA;

hall Aerospace \& Technologies Corp., Boulder CO, USA; iThe Pennsylvania State University, University Park, PA 16802, USA.; jSpace Sciences Lab., Univ. of California, Berkeley, USA;

\begin{abstract}
The long-term stability of exoplanetary atmospheres depends critically on the extreme-ultraviolet (EUV) flux from the host star. The EUV flux likely controls the demographics of the short-period planet population as well the ability for rocky planets to maintain habitable environments long enough for the emergence of life. We present the Extreme-ultraviolet Stellar Characterization for Atmospheric Physics and Evolution (ESCAPE) mission, an astrophysics Small Explorer proposed to NASA. ESCAPE employs extreme- and far-ultraviolet spectroscopy (70 - $1800 \AA$ ) to characterize the highenergy radiation environment in the habitable zones (HZs) around nearby stars. ESCAPE provides the first comprehensive study of the stellar EUV environments that control atmospheric mass-loss and determine the habitability of rocky exoplanets. The ESCAPE instrument comprises an EUV grazing incidence telescope feeding four diffraction gratings and a photon-counting detector. The telescope is $50 \mathrm{~cm}$ diameter with four nested parabolic primary mirrors and four nested elliptical secondary mirrors, fabricated and aligned by NASA Marshall Space Flight Center and the Smithsonian Astrophysical Observatory. The off-plane grating assemblies are fabricated at Pennsylvania State University and the ESCAPE detector system is a micro-channel plate (MCP; $125 \mathrm{~mm} \times 40 \mathrm{~mm}$ active area) sensor developed by the University of California, Berkeley. ESCAPE employs the versatile and high-heritage Ball Aerospace BCP-100 spacecraft.
\end{abstract}

Keywords: stars, exoplanets, extreme-ultraviolet, spectroscopy, Explorer mission

\section{INTRODUCTION}

With approximately 4,000 confirmed extrasolar planets known, our next key challenge is to explore the environments that can support life. Habitable planets, as currently defined, are those with an atmosphere able to sustain liquid water over several billion years. The concept of the habitable zone (HZ) was created with the solar system in mind because our experience is that a rocky planet can evolve into a habitable world at 1 astronomical unit from a solar-type star. However, the planetary effective surface temperature alone is insufficient to characterize the habitable zone. The stellar bolometric luminosity provides insufficient information to accurately predict and interpret biosignature gases (Segura et al. 2005; Hu et al. 2012; Tian et al. 2014 ; Harman et al. 2015; Meadows et al. 2018) or evaluate the potential for rocky planets to

UV, X-Ray, and Gamma-Ray Space Instrumentation for Astronomy XXI, edited by Oswald H. Siegmund, Proc. of SPIE Vol. 11118, $1111808 \cdot$ C 2019 SPIE · CCC code: 0277-786X/19/\$21 · doi: 10.1117/12.2526859 


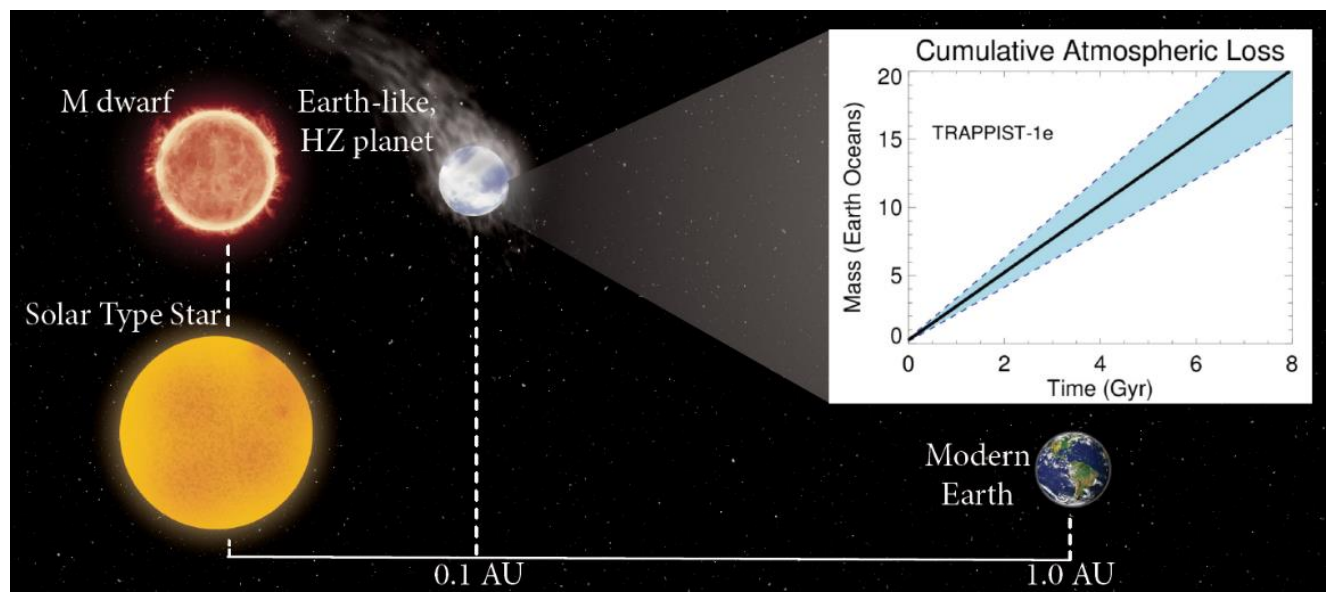

Figure 1. - Temperate, rocky planets around $\mathrm{M}$ dwarfs may rapidly lose $10-20$ Earth oceans (or 2,500 - 5,000 1 bar atmospheres) over their lifetimes their atmosphere due to high stellar EUV fluxes in the HZ. The inset shows a mass-loss simulation for the most promising HZ planet in the Trappist-1 system (shaded region are uncertainties from FUV observations; Bourrier et al. 2017). ESCAPE provides the stellar EUV input for understanding habitable exoplanetary atmospheres.

maintain habitable conditions (Lammer et al. 2009; Airapetian et al. 2017). The stellar high-energy radiation environment is the single largest forcing function for long-term habitability of exoplanet atmospheres (Figure 1). One of the major questions for astronomy in the next three decades is what fraction of temperate, rocky planets around stars of different types can develop and retain a habitable atmosphere?

NASA's current astrophysics MidEx mission (TESS), the flagship missions of the 2020s (JWST), and all four of the large mission concept studies being carried out ahead of the 2020 Astrophysics Decadal Survey (LUVOIR, Habex, OST, and Lynx) have the detection or characterization of habitable planets as part of their primary science goals. However, none of these missions will observe the primary driver for atmospheric physics and evolution: the EUV radiation (Section 2.1) and particle environments (Section 2.2) imposed by their host stars. ESCAPE provides the stellar EUV context for models of exoplanet habitability and provides a bridge between NASA's previous/existing exoplanet detection and characterization missions and future life-finder missions by providing a roadmap to the most promising systems for investment of resources and observing time.

\section{THE STABILITY AND EVOLUTION OF HABITABLE ATMOSPHERES}

\subsection{EUV Influences and the ESCAPE SEEN Survey}

The long-term habitability of an atmosphere is determined by the flux of volatiles from the surface to the top of the atmosphere. Different parts of a star's spectral energy distribution (SED) and particle output drive heating and chemistry in different layers of a planet's atmosphere due to the wavelength dependence of atomic and molecular photoabsorption cross sections. Optical and near-infrared photons heat the surface and troposphere. NUV (1800 - 3200 ^), FUV (912 $1800 \AA$ ), and X-ray $(5-100 \AA$ ) photons are absorbed in the middle and upper atmosphere where they photodissociate molecules. EUV photons (100-911 $⿱$ A) are absorbed high in the atmosphere (i.e., in the thermosphere) where they ionize atoms and molecules. Liberated electrons collisionally heat the surrounding gas, increasing the scale height of the atmosphere and potentially leading to the formation of a hydrodynamic outflow (i.e., rapid atmospheric escape).

EUV photons are the key drivers for atmospheric mass-loss for two primary reasons: 1) EUV photons are absorbed in the highest (lowest density) layers of the atmosphere, where radiative losses (proportional to density squared) are minor and the heating efficiency is highest, and 2) there are many more EUV photons than X-rays available on all types of stars to drive this heating. In the quiet Sun, the EUV/X-ray photon production ratio is $\sim 90$ (Woods et al. 2009). For optically inactive early M dwarfs the EUV/X-ray photon ratio is $\sim 40$ (Fontenla et al. 2016).

The discovery of rocky planets in the HZs of nearby M dwarfs, e.g., Proxima Cen b and the TRAPPIST-1 planets, have motivated new atmospheric mass loss calculations that highlight the need for improved EUV irradiance data to estimate 


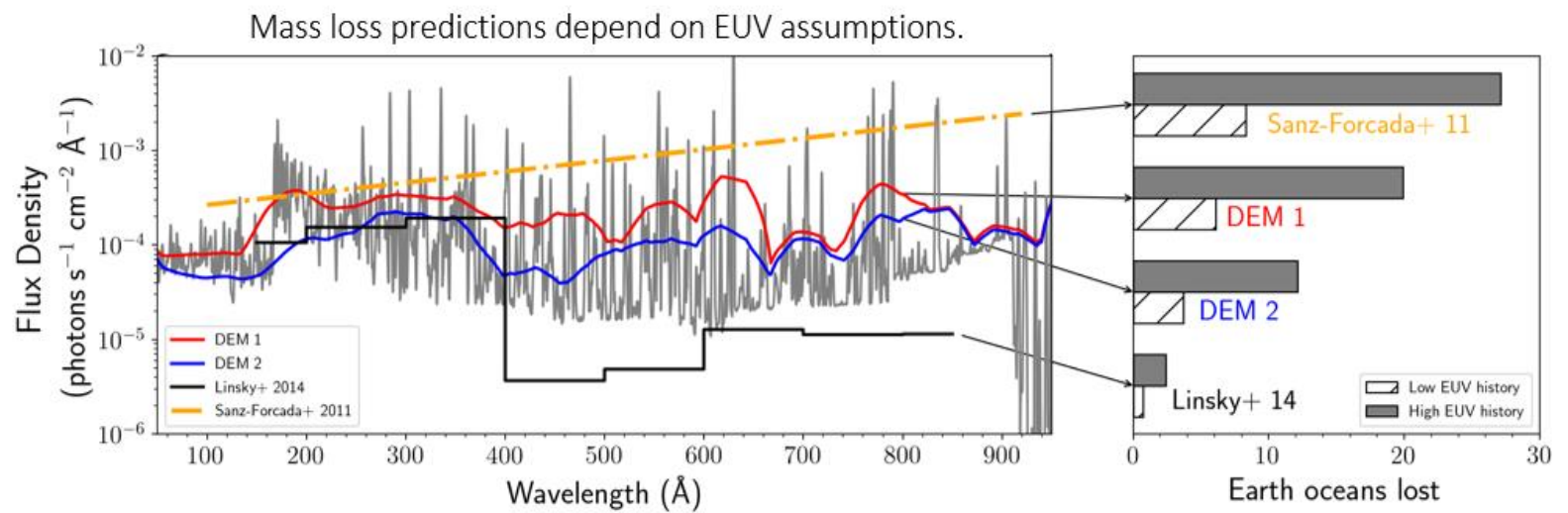

Figure 2 - (left) Different reconstructions of the EUV spectrum of the M dwarf Proxima Cen show factors of 3-100 flux discrepancies: differential emission measure models in red, blue, and gray (Drake et al. 2019); scaling relations based on only FUV in black (Linsky et al. 2014) and only X-rays in orange (Sanz-Forcada et al. 2011). Corresponding hydrogen mass lost from an Earth-like planet (in units of Earth oceans) from 10 Myr to $4.8 \mathrm{Gyr}$ is shown at right for high (shaded gray) and low (hatched) EUV histories of typical M dwarfs (Ribas et al. 2017). ESCAPE resolves the factor of $~ 30$ differences in atmospheric mass loss rates by directly measuring the EUV spectrum to $40 \%$ accuracy. ESCAPE spectra are inputs for atmospheric loss models of terrestrial planets orbiting stars of different ages and masses.

their long-term habitability (Figure 2). Garcia-Sage et al. (2017) presented a study of EUV-driven proton and $\mathrm{O}^{+}$escape from an Earth-like planet orbiting Proxima Cen. They found mass loss rates several orders of magnitude higher than present-day Earth for EUV fluxes 10 - 20 times the current day EUV solar irradiance. This escape results in complete atmospheric loss in less than $0.5 \mathrm{Gyr}$, even in the presence of a terrestrial-strength magnetic field. A factor of ten in the uncertainty of incident EUV flux can lead to three orders of magnitude in the uncertainties of ion escape rates, fundamentally shifting our predictions for which exoplanets are the best candidates for long-lived habitable atmospheres. Airapetian et al. (2017) reached similar conclusions on the loss of one bar of $\mathrm{H}$ and $\mathrm{O}$ via ion escape. Their models indicate that elevated EUV fluxes from persistent flares or the long pre-main-sequence phase of M dwarfs could effectively render some Earth-like planets around M dwarfs barren in the absence of internal or external resupply of volatiles. However, the EUV luminosity, flare rate, and evolution of $\mathrm{M}$ dwarfs are almost completely unconstrained. These quantities can only be empirically confirmed with direct measurements of the absolute flux and temporal variability of a sample of stars with a range of masses, ages, and activity levels.

ESCAPE directly measures the absolute flux and time-evolution of EUV irradiance from nearby stars to explore the physics and evolution of potentially habitable worlds, and identify the most promising exoplanetary systems for atmospheric spectroscopy in the next two decades. To set the conditions for the stability of exoplanetary atmospheres, we require sufficient sensitivity to reach a statistical sample of F, G, K, and M stars - the Stellar Euv ENvironments (SEEN) survey measures the $70-1800 \AA$ irradiance and time variability for approximately 200 stars with absolute photometric uncertainty $<40 \%$ in a nominal 2 year mission. We require the sensitivity and spectral resolution to separate stellar emission lines and measure variability in individual spectral features. These requirements drive the telescope size and grazing incidence design to maintain high throughput at EUV wavelengths. FUV spectra are formed in same region of the stellar atmosphere as longer-wavelength $(570-911 \AA)$ EUV emission; FUV spectra enable us to reconstruct that flux when it is inaccessible due to interstellar attenuation. FUV spectra are acquired simultaneously to constrain calculations of the complete EUV energy budget due to the time-variability of the EUV and FUV bands (Loyd et al. 2018a,b). The 570 - $911 \AA$ flux estimates are validated by direct observation of the $600-810 \AA$ flux from the nearest stars, providing a robust empirical basis for the complete EUV irradiance measured by ESCAPE, and defining the spectral coverage requirements.

\subsection{Particle Influences and the ESCAPE DEEP Survey}

High-energy particles have a major influence on the evolution of planetary atmospheres, both through the actions of stellar winds and impulsive events (e.g., coronal mass ejections, CMEs). While stellar winds play a part in atmospheric escape, CMEs are thought to be the dominant source of long-term instability in planetary atmospheres (Khodachenko et al. 2007; Lammer et al. 2018; Cherenkov et al. 2017). The influence of CMEs depends strongly on their frequency and ability to break out of coronal magnetic confinement; ESCAPE measures the frequencies of CMEs on solar-type stars and search 
for CME breakout from M dwarfs. While the main source of heat in the upper atmosphere of planets is the EUV flux, joule heating and particle precipitation heating could dominate the chemical impact of impulsive events (e.g., Segura et al. 2010; Tilley et al. 2018). CMEs deliver heat to the upper atmospheres of orbiting planets through charge exchange reactions (Chassefiere 1996). CMEs contribute to atmospheric mass loss on solar system planets and to space weather, leading several authors to propose the "space weather habitable zone" (e.g., Airapetian et al. 2017) as more meaningful than the traditional liquid water HZ.

Approximately $90 \%$ of large (X-class) solar flares are associated with CME-like particle eruptions, however, this connection has not been borne out with recent observations of stellar flares (Osten \& Wolk 2015, Odert et al. 2017, Crosley \& Osten 2018). While the EUV irradiance is increased by a large flare rate, CMEs and accelerated particles may have much greater impacts on atmospheric photochemistry and stripping than the flare radiation itself (Segura et al. 2010; Tilley et al. 2018; Lammer et al. 2007).

For the Sun, we are able to observe CMEs directly with coronagraphs and in situ measurements. These traditional methods are not feasible for stellar CMEs in the near-term (although see Haisch et al. 1983 and Moschou et al. 2017). However, a number of other techniques for detecting solar CMEs have been developed. Harra et al. (2016) reviewed these techniques and determined that coronal dimming, described below, is the only feature consistently associated with CMEs that can be employed to detect and quantify these events on other stars.

Solar coronal dimming studies characterize the transient voids left behind in the corona as a CME departs, i.e., the observed flux dimming (e.g., Sterling and Hudson 1997, Aschwanden et al. 2009, Reinard and Biesecker 2009; Dissauer et al. 2018a, b). The CU-led Solar Dynamics Observatory EVE instrument (SDO/EVE; Woods et al. 2012) demonstrated that dimming can also be characterized with disk-integrated EUV spectra (Woods et al. 2011, Mason et al. 2016, 2019a). ESCAPE employs the same 'Sun-as-a-star' stellar CME characterization techniques validated on SDO/EVE (Figure 3) to measure the frequency of CMEs on nearby stars for the first time.

The dimming light curve also contains information about the kinematics of the CME that produced it. Solar dimming lightcurves have been calibrated by in-situ proton measurements with the GOES satellites. The depth of the dimming event is directly related to the mass of the ejected CME (the dispersal of the quiescent corona greatly reduces the EM, which scales as density squared), whereas the slope of the dimming lightcurve indicates the CME propagation velocity (Mason et al. 2016, 2019b). Therefore, observations of coronal dimming events on solar-type stars can provide direct constraints on the kinetic energy of CME events (mass $\times$ velocity ${ }^{2}$ ).

The Dedicated Euv Eruption Program (DEEP) survey executes monitoring campaigns of 24 select stars to measure EUV flare frequency distributions and characterize stellar CMEs for the first time. The DEEP survey monitors stars for
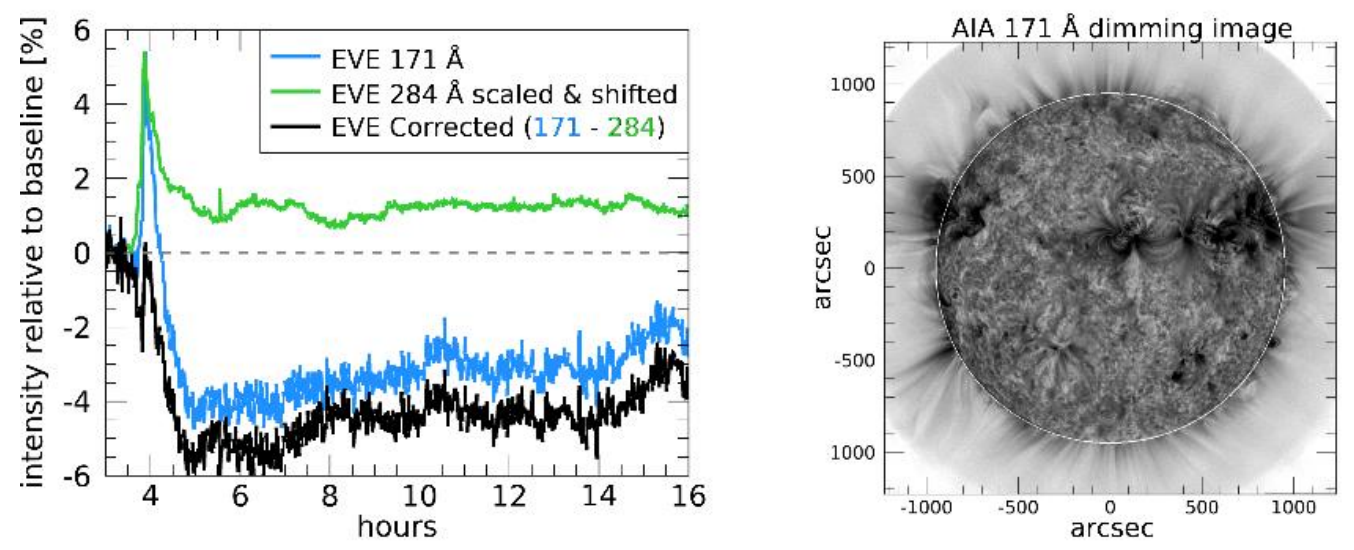

Figure 3 - Observation of coronal dimming in full-disk solar spectra (left) and angularly resolved filter imaging (right). The spectrally-resolved lightcurves from CU/LASP's SDO-EVE show flaring lines (Fe XV $284 \AA$, green), dimming lines (Fe IX $171 \AA$ A, blue), and the corrected Fe IX $171 \AA \AA$ dimming profile in black (Mason et al. 2016). ESCAPE is sensitive to similar events on F, G, and $\mathrm{K}$ dwarfs to a distance of $5 \mathrm{pc}$. 66 candidate events are expected in the DEEP survey. 


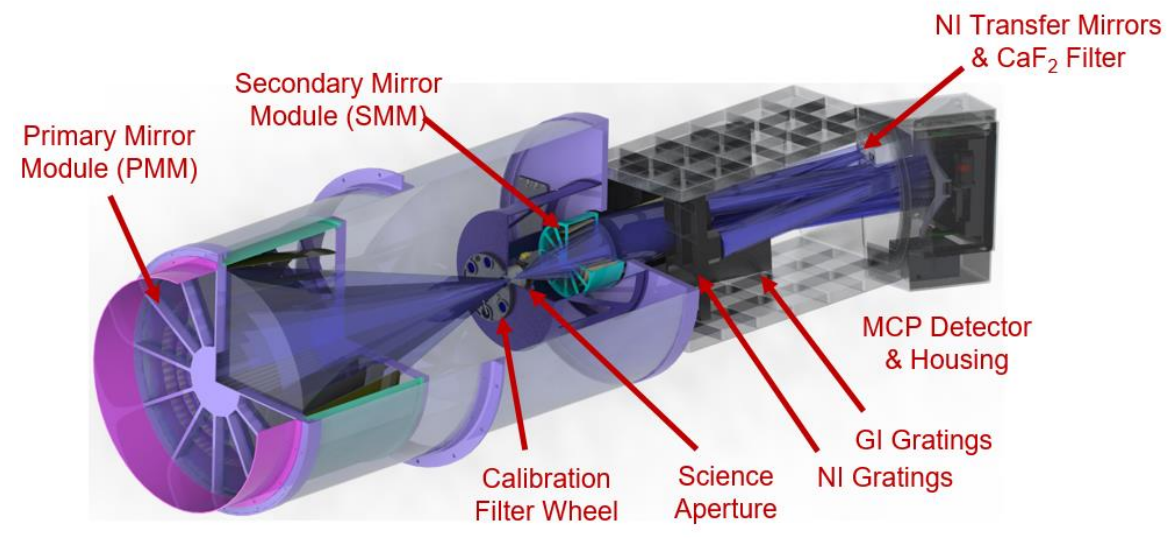

Figure 4: Instrument Overview - Shaded CAD model of the ESCAPE instrument with overlaid ray cones. Instrument modules are labeled. Major instrument components are labeled.

sufficient duration ( 2 weeks) to measure (or place stringent constraints on) coronal dimming associated with CMEs. ESCAPE has the sensitivity and spectral coverage to detect large solar-like dimming events to a distance of 5 pc. ESCAPE will survey $10 \mathrm{~F}, \mathrm{G}$, and $\mathrm{K}$ stars for solar-like CMEs as part of the DEEP survey.

\section{ESCAPE INSTRUMENT AND SPACECRAFT IMPLEMENTATION}

EUV spectra of exoplanet host stars are scarce, particularly for M dwarfs. The only previous EUV astronomy mission, EUVE (Bowyer et al. 1991), obtained spectra of $\sim 15$ cool main sequence stars, including $5 \mathrm{M}$ dwarfs. Previous observations were heavily biased toward the most active stars. The very modest $\leq 2 \mathrm{~cm}^{2}$ effective area of the EUVE spectrometers precluded statistical studies of stars of a range of mass and age. ESCAPE's sensitivity requirement corresponds to > 50x the effective area of EUVE across the 100-500 $\AA$ bandpass, which drives the size and efficiency of the optical system elements. ESCAPE exceeds this requirement with over 100\% margin by combining technologies developed and flight qualified since EUVE with a $50 \mathrm{~cm}$ diameter grazing incidence Hettrick-Bowyer Type I telescope (hereafter HB; Hettrick \& Bowyer, 1984). The telescope feeds a single instrument with four simultaneous EUV/FUV spectroscopic channels (Figurer 4).

\subsection{Telescope}

The HB is the grazing incidence equivalent of a Gregorian telescope: a parabolic primary and an elliptical secondary mirror with a prime focus in between (Figures 4 and 5). ESCAPE consists of four nested, confocal parabola-ellipse pairs to increase collecting area relative to a single telescope. The HB is the optimum design for ESCAPE for two primary reasons. First, a $1.5 \mathrm{~mm}$ diameter aperture at prime focus (effective width: 3.1', including aperture losses) reduces the geocoronal $\mathrm{H} \mathrm{I}, \mathrm{He}$ I, and He II background flux into the instrument by limiting the field-of-view subtended by the science aperture, without introducing the throughput reduction associated with band-shaping filters. This suppresses the sky background

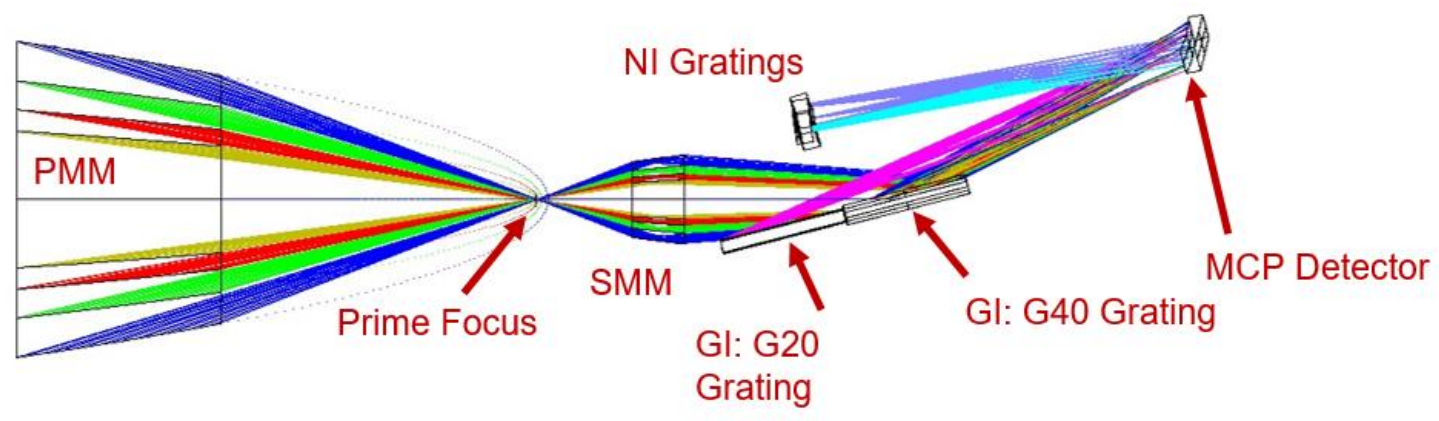

Figure 5: ESCAPE Science instrument raytrace. PMM and SMM are the telescope primary and secondary mirror assemblies. The GI and NI are grazing incidence and normal incidence spectrograph systems, respectively. 
flux entering the instrument by factors of $>250$ relative to EUVE (Boyd et al., 1994). Second, the ESCAPE telescope is less complex and has higher throughput in the EUV, as EUV photons do not require as shallow of graze angles as X-rays, than would scaled-up diameter and focal length versions of existing X-ray imaging spectrographs, such as the five-optic MaGIXS instrument (Kobayashi et al., 2018).

The optics for ESCAPE are manufactured at NASA MSFC via electroformed nickel replication (ENR; see e.g. Ramsey 2006), the same optic fabrication technique used for the Imaging X-ray Polarimetry Explorer (IXPE), XMM-Newton (Jensen et al., 1989), as well as the Heliophysics sounding rockets FOXSI and MaGIXS. ENR optics are thin, lightweight, and rigid, and therefore compatible with a nested configuration to increase collecting area.

Each of the four telescope units (hereafter HB Shells 1-4, consisting of Primary Shells 1-4 and Secondary Shells 1-4) have a focal length of $3.1 \mathrm{~m}$. The largest shell (Primary Shell 1) has an outer diameter of $50 \mathrm{~cm}$, while the innermost shell (Primary Shell 4) has an inner diameter of $16.8 \mathrm{~cm}$. The platescale is $66^{\prime \prime} / \mathrm{mm}$ and the projected RMS diameter of the resulting spots is $34.3^{\prime \prime}$. This is comparable to other grazing incidence telescopes, including IXPE ( $36^{\prime \prime}$ RMS, inproduction, ENR optics; O’Dell et al., 2018), XMM-Newton ( 22" RMS, ESA, ENR optics; Gondoin et al., 1998) and NuStar ( 60" RMS, segmented glass; Koglin et al., 2016). HB Shells $1 \& 2$ are coated with platinum (Pt) while HB Shells $3 \& 4$ are coated with zirconium $(\mathrm{Zr})$. $\mathrm{Zr}$ has $\sim 80 \%$ reflectivity in the $140-220 \AA$ band at the shallower reflection angles of Shells $3 \& 4$, while Pt maintains higher broadband reflectivity at the steeper $\theta$ 's of Shells $1 \& 2$.

\subsection{Spectrograph}

ESCAPE features four gratings, all of which have a fixed position, operate simultaneously, and disperse light onto different locations on the same science detector. The two grazing incidence (GI) gratings (G20 and G40) intercept different portions of the telescope beam prior to HB focus and disperse light directly onto the detector. This is similar to the DS/S on EUVE, which fed six different fixed position spectrographs and imagers from the same telescope (Sirk et al., 1996). The two normal incidence (NI) channels pickoff the zero-order reflections of the GI gratings and direct long-wavelength EUV (G70) and FUV (G140) spectra back onto the science detector.

The GI gratings intercept $\sim 3 \%$ (G20) and 57\% (G40) of the telescope beam at an average graze angle of $\theta=14.4^{\circ}$ (Figure $5)$ and are similar to radial off-plane gratings where the grooves are blazed and patterned quasi-parallel to the converging rays (Miles et al. 2018; McEntaffer et al., 2019). The ESCAPE gratings deviate from a perfect radial pattern due to a small amount of groove curvature for aberration correction. Light is diffracted along an "arc of diffraction" into curved spectra. The bandpass and spectral resolution of each channel are enhanced by using multiple spectral orders down to the short wavelength limit of $\lambda>65 \AA$ set by the reflectivity of the optics.

\begin{tabular}{|c|c|c|c|c|c|c|c|c|}
\hline & $\mathrm{G} 20$ (GI-S) & G40 (GI-L) & Notes & & & & & \\
\hline Bandpass & $65-305 \AA$ & $65-568 \AA$ & Multiple & & & & & \\
\hline Blaze $\lambda$ & $180 \AA$ & $360 \AA$ & orders & & & & & \\
\hline 1st Order Bandpass & $104-305 \AA$ & $250-565 \AA$ & Detector Limit & \multirow{2}{*}{\multicolumn{5}{|c|}{ Table 2: Calibration Filter Wheel Elements }} \\
\hline 1st Order Resolution & 0.84 & 1.34 & $\Delta \AA$ & & & & & \\
\hline 2nd Order Bandpass & $65-153 \AA$ & $125-283 \AA$ & Detector Limit & Position & Material & Bandpass & G20 Orders & G40 Orders \\
\hline 2nd Order Resolution & 0.42 & 0.67 & $\Delta \AA$ & $1 \& 4$ & $\mathrm{Zr}+\mathrm{MoSi}_{2}$ & $100-200 \AA$ & $m=1$ & $m=2,3$ \\
\hline 3rd Order Bandpass & $65-102 \AA$ & $83-188 \AA$ & $A_{\text {eff Limit }}$ & $2 \& 5$ & $\mathrm{Al}+\mathrm{Mg}$ & $250-560 \AA$ & $m=1$ & $m=1$ \\
\hline $\begin{array}{l}\text { 3rd Order Resolution } \\
\text { 4th Order Bandpass }\end{array}$ & $\frac{0.28}{--}$ & $\frac{0.45}{65-141 \AA}$ & $\frac{\Delta \AA}{A_{\text {off }} \text { Limit }}$ & $3 \& 6$ & Indium & $<120 \AA$ & $m=2$ & $m=4$ \\
\hline 4th Order Resolution & - & 0.33 & $\Delta \AA$ & 7 & Open & $70-560 \AA$ & All & All \\
\hline \multicolumn{4}{|c|}{ ESCAPE Normal Incidence Spectrograph Parameters } & & & & & \\
\hline & G70 (NI-S) & G140 (NI-L) & Notes & & & & & \\
\hline Bandpass & $600-825 \AA$ & $1280-1800 \AA$ & 1st Order & & & & & \\
\hline Blaze $\lambda$ & $700 \AA$ & $1400 \AA$ & Only & & & & & \\
\hline Resolution & 3.60 & 6.10 & $\Delta \AA$ & & & & & \\
\hline
\end{tabular}

Each GI grating has a ruled area of $198 \times 115 \mathrm{~mm}$. The bandpass and resolution of the first three spectral orders is shown in Table 1. The effective areas of orders $\mathrm{m}>3$ for the G20 and $\mathrm{m}>4$ for the G40 do not exceed $1 \mathrm{~cm}^{2}$ for any wavelength. The G20 channel is optimized for short wavelengths and coated with $\mathrm{Zr}$, while the G40 is optimized for a broader bandpass and coated with Pt. The ESCAPE gratings are lithographically ruled into single crystal silicon with exceptionally smooth groove profiles (Miles et al., 2018). The precision of this ruling method reduces scatter and increases efficiency by several factors relative to the gratings on EUVE. Gratings ruled in this manner are TRL-6-7 for X-ray spectroscopy. 
A 1" test grating matching an early prescription of the G20 grating was fabricated at the Nanofabrication Lab at Penn State University (PSU). This test grating was characterized at the Advanced Light Source synchrotron facility following the established procedure outlined in Miles et al., 2018. The peak diffraction efficiency of this test-grating was measured to be $77 \%, \sim 25 \%$ greater than the peak efficiency used to calculate the ESCAPE effective area (Figure 6). The ESCAPE team has elected to hold this as an effective area reserve in favor of a more conservative peak efficiency of $62 \%$ for performance projections.
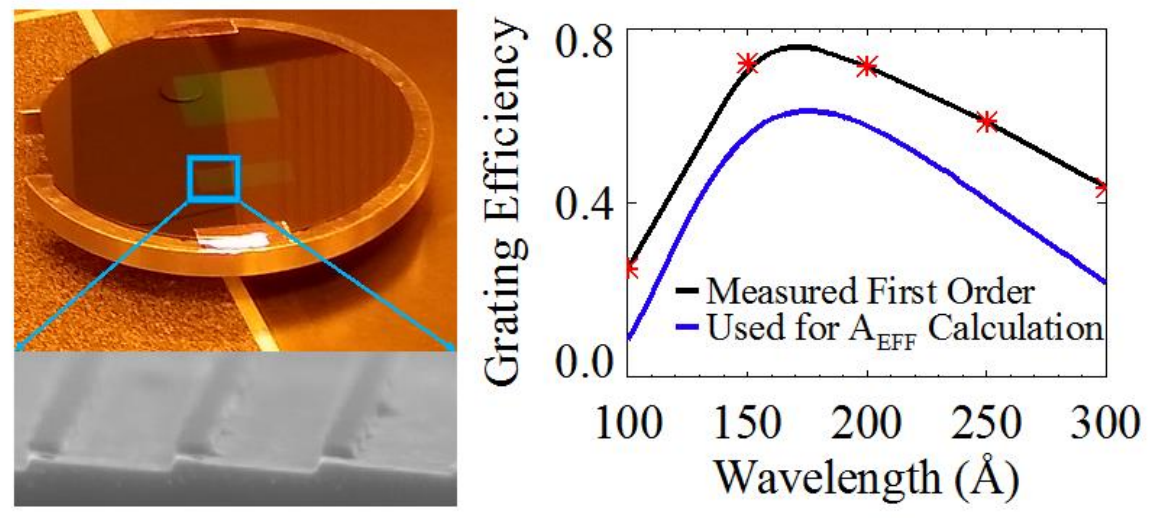

Figure 6: (Left) The 1" test grating fabricated for efficiency measurements and TRL advancement, with an SEM image of the blazed grooves from a practice etch. (Right) Measured diffraction efficiency of the test piece (red stars) relative to the curve used to calculate the ESCAPE effective area.

The normal incidence channels provide complimentary bandpass coverage of the long wavelength EUV (600 - 810 Å; G70) and FUV (1300 - $1800 \AA$ § G140). These channels add minimal additional complexity by leveraging the otherwise wasted zero-order reflection of the GI gratings. The G70 channel is fed by zero-order G20 with both the grating and fold mirror coated in silicon carbide. The G140 channel is fed by zero-order G40 (the two pickoff points are separated by $\sim 27$ $\mathrm{mm}$ ) with $\mathrm{MgF}_{2}$ protected aluminum coatings. $\mathrm{A} \mathrm{CaF}_{2}$ filter in the $\mathrm{G140}$ optical path removes $>99 \%$ of geocoronal $\mathrm{H} \mathrm{I}$ Ly $\alpha$ to reduce scattered light background. A bi-stable shutter blocks the G140 aperture when ESCAPE observes FUVbright targets. The NI gratings will be ruled using the same technique at PSU, however on a concave substrate.

\subsection{Detector System}

The four ESCAPE gratings image spectra onto a cylindrically-curved $(\mathrm{R}=0.5 \mathrm{~m}), 125 \times 40 \mathrm{~mm}$ atomic layer deposited (ALD), borosilicate glass MCP, cross delay line (XDL) readout detector with a KBr photocathode for optimal EUV QE. MCP-based detectors have been used on many EUV and FUV missions (GALEX, EUVE, FUSE, HST-COS; Green, 2012) because of their low background rate (photon-counting with no read noise), high in-band quantum efficiency (QE; Siegmund et al., 1988), and configurability for many focal plane sizes and shapes. The detector and readout electronics will be implemented by the University of California based on extensive flight mission heritage. Current XDL detectors (e.g., McCandliss et al., 2016) regularly achieve spatial resolutions $<60 \mu \mathrm{m}$ FWHM with global rate capabilities in excess of $400 \mathrm{kHz}$ and have demonstrated excellent gain stability (Siegmund et al. 2017).

On-orbit background is determined by charged particle and gamma-ray events in addition to intrinsic background (Siegmund et al., 1989). The borosilicate substrate of the ALD-MCPs (Siegmund et al., 2015, Ertley et al., 2015) reduces the overall on-orbit LEO background rate $\left(<0.6 \mathrm{~cm}^{-2} \mathrm{sec}^{-1}\right.$ in orbit radiation, $\sim 0.05 \mathrm{~cm}^{-2} \mathrm{sec}^{-1}$ intrinsic) relative to conventional MCPs $\left(\sim 0.5 \mathrm{~cm}^{-2} \mathrm{sec}^{-1}\right.$ intrinsic from ${ }^{40} \mathrm{~K}$ beta decay; Vallerga et al., 2001). A background coincidence shield is included to reduce the particle background rate by $\geq 70 \%$ (demonstrated on the UCB-led EURD mission; Bowyer et al., 1997), enabling low background $\left(<0.25 \mathrm{~cm}^{-2} \mathrm{sec}^{-1}\right)$ for detection of faint targets.

\subsection{Order-sorting and EUV Narrow-band Filters}

Spectral order calibration is performed during $35 \mathrm{ksec}$ exposures on bright sources of different spectral type/coronal temperature as part of our standard calibration program. The ESCAPE order-sorting filters are provided by Luxel and are similar to those flying on the CU-led SDO-EVE instrument (Table 2). The Al/Mg filter isolates the first order G40 spectrum (and enables the calculation of a predictive DEM model to guide the extraction). The 'Open $-\mathrm{Al} / \mathrm{Mg}$ ' residual 


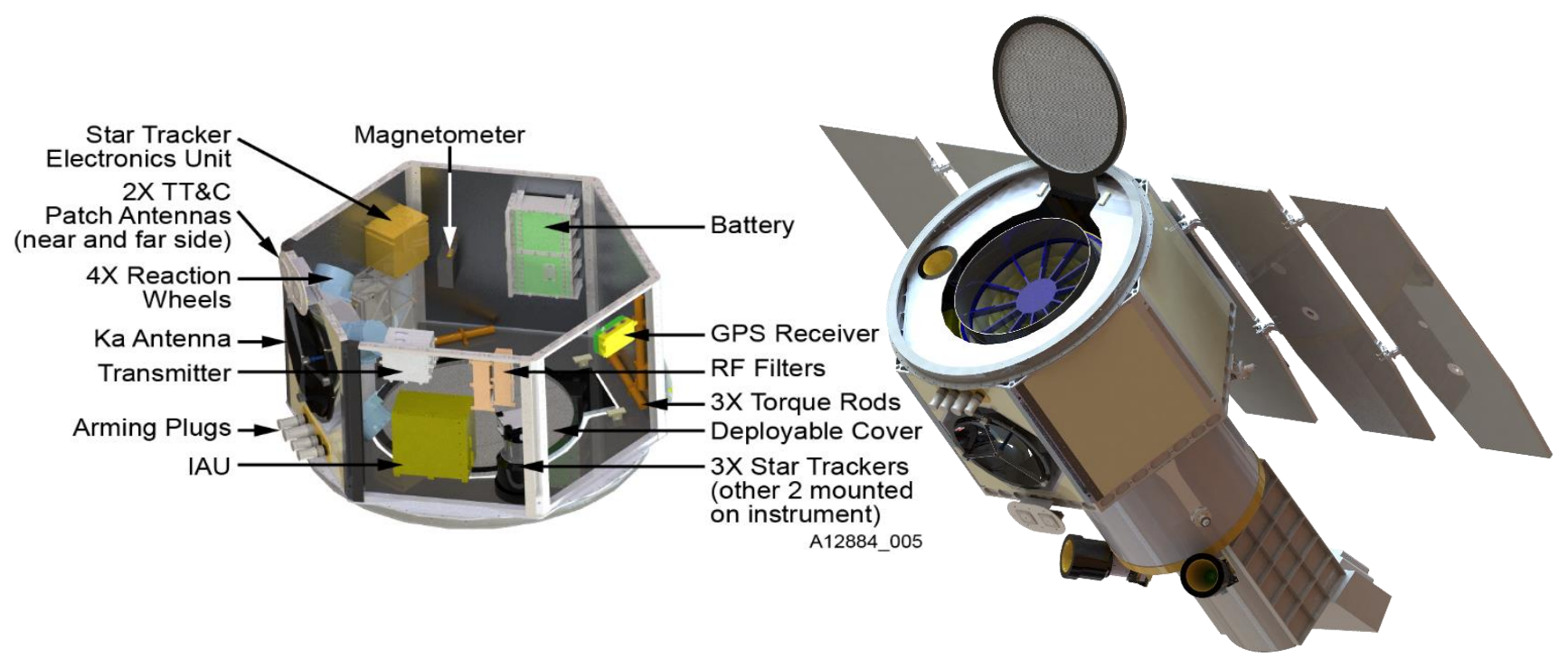

Figure 7 - (left) The ESCAPE spacecraft is derived from the Ball BCP line with high-heritage components from previous missions. (right) Integrated ESCAPE spacecraft and science instrument in deployed, on-orbit configuration.

G40 spectra are cross-correlated with the $\mathrm{Zr} / \mathrm{MoSi}$ G20 spectra to isolate the first and second order G20 and G40 spectra. The In filter isolates fourth order light and controls for short-wavelength bremsstrahlung leaking into the ESCAPE band.

The order-sorting calibration filter wheel (FW; Figure 4) consists of an 8-position filter wheel located $15 \mathrm{~mm}$ offset from prime focus. The FW is nominally at an OPEN setting with no filter in place for all SEEN and DEEP observations. The other FW positions contain primary and backup filters provided by Luxel and listed in Table 2. These filters are $10 \mathrm{~mm} x$ $10 \mathrm{~mm}$ and supported on an electroformed nickel mesh ( $87 \%$ transmission) to ensure launch vibration survival.

\subsection{Spacecraft}

The ESCAPE spacecraft is derived from the Ball BCP smallsat line, which was flight demonstrated with the USAF STPSat-2 and STPSat-3 missions. ESCAPE's spacecraft is based on the GPIM mission bus, successfully launched to a LEO orbit and commissioned in June 2019. The spacecraft bus is also in development for NASA's IXPE SMEX and SPHEREx MIDEX missions. These five missions are low Earth, Sun synchronous observatories of similar size and requirements. The spacecraft is a single-string architecture with functional redundancy in safe mode using back-up ACS components (coarse sun sensors and 3-axis magnetometer, Figure 7). The integrated spacecraft and instrument in deployed configuration is shown in Figure 7.

Predicted pointing stability (driven by ESCAPE's $1.5 \AA$ A spectral resolution requirement) is derived from a simulation that incorporates star tracker performance and observatory control system characteristics. The simulation assumes one of the three star tracker heads is obscured as a worse case assumption. Simulation results demonstrate that LoS pointing stability meets the 8 " spacecraft stability requirement with $54 \%$ margin and the 300 s pointing stability interval is the time required to build up a trackable stellar signal to correct drifts for a typical SEEN survey target. The ESCAPE simulation results are validated by WISE on-orbit demonstration of similar performance.

\section{INSTRUMENT PERFORMANCE PREDICTIONS AND SCIENCE PROGRAM}

\subsection{ESCAPE Effective Area and Signal-to-Noise Estimates}

The effective area for each channel is determined by geometrically modelling the graze angle of rays off each surface and then determining the reflection efficiency for the appropriate mirror coatings at those angles. The resulting throughput is multiplied by the grating diffraction efficiencies, detector quantum efficiency and any other intervening elements. The grating efficiency is modeled and includes the effect of phase interference and blaze shadowing, and then scaled to a peak efficiency of $62 \%$ in first order as an average value for a typical blazed grating. This is $~ 25 \%$ less than the measured efficiency for the ESCAPE test sample produced for this proposal (Figure 6). The difference is held as effective area 

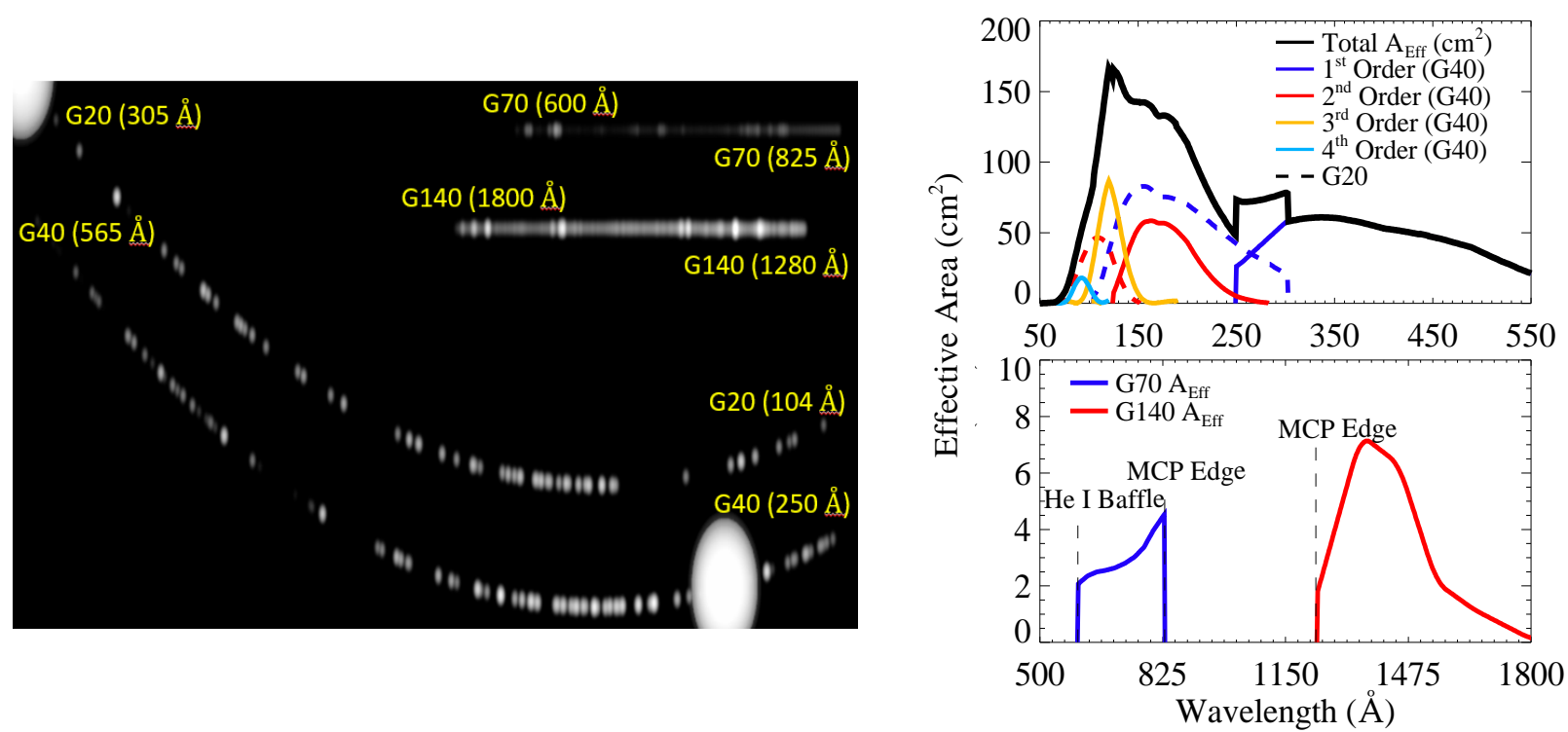

Figure 8 - (left) Simulated ESCAPE focal plane image; aspect ratio is compressed 2:1 for display. This spectrum represents an $8 \mathrm{MK}$ coronal plasma, with the brightness of the M star AU Mic. He II $304 \AA$ airglow are the large spots. Detector background of 0.25 counts $\mathrm{cm}^{-2} \mathrm{~s}^{-1}$ is included, but is much lower than the source flux. (right) Effective areas $\left(\mathrm{A}_{\mathrm{Eff}}\right)$ for the ESCAPE channels, taking into account the reflection geometry of the optics, groove shadowing as a function of position on the grating diffraction efficiency.

reserve and all performance calculations use the more conservative model estimate. The effective area curves for all modes, as well as simulated two-dimensional spectra are given in Figure 8.

$\mathrm{S} / \mathrm{N}$ estimates are calculated for 485 candidate targets. Stellar fluxes are estimated from archival data, DEM calculations, or scaling relations (Linsky et al. 2014; Sanz-Focada et al. 2011; Youngblood et al 2017; France et al. 2018). Fluxes are "reddened" before calculating SC by computing optical depth spectra for $\mathrm{H}, \mathrm{He}$, and $\mathrm{He}^{+}$(France et al. 2018). Elements heavier than He do not contribute appreciably to the LISM opacity at $\lambda>100 \AA$ (Morrison \& McCammon 1983). EUV fluxes are converted to spectral estimates for every target by assuming the EUVE SED of $\alpha \mathrm{Cen} A+\mathrm{B}$, a star system whose spectra display lines of both low and intermediate coronal gas temperatures. The resultant emission line fluxes are used to

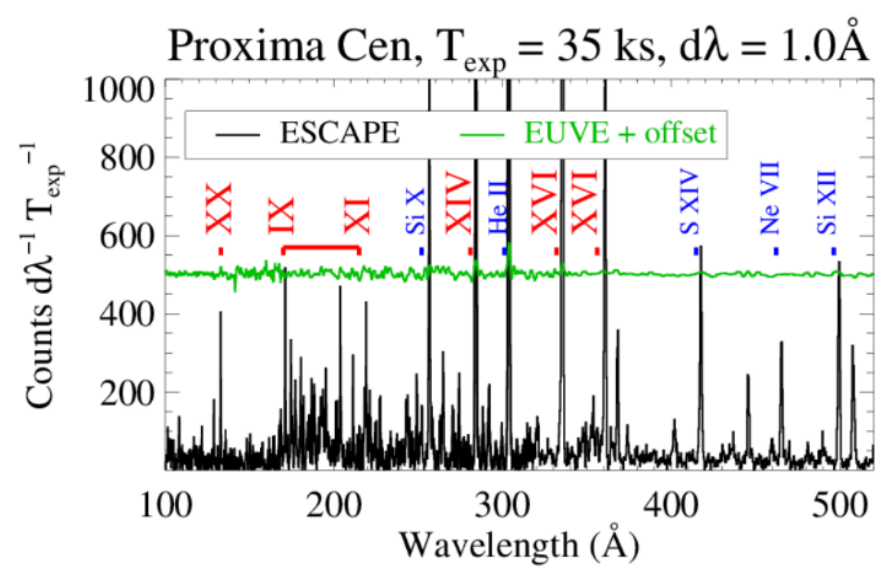

Figure 9 - ESCAPE's $\sim 100 x$ gain in sensitivity over EUVE (A $A_{\text {eff }}(E U V E) \leq 2 \mathrm{~cm}^{2}$ at peak) allows it to survey stellar EUV emission for the first time. A simulated ESCAPE spectrum of Proxima Cen is shown (black). The $77 \mathrm{ks}$ EUVE exposure of Prox Cen (green, offset by 500 counts) resulted in tentative detections of $\sim 5$ stellar emission lines. ESCAPE achieves a line-integrated S/N of $\sim 35$ at Fe IX $171 \AA$ in $35 \mathrm{ksec}$. Prominent iron ionization states are labeled in red and other species in blue. 
estimate the $\mathrm{S} / \mathrm{N}$, providing an estimate of the quality of the resultant spectrum, without requiring precise knowledge of the shape of the stellar SED.

We present an example $\mathrm{S} / \mathrm{N}$ calculation for the DEM calculated spectrum of Proxima Cen (Figures 9): At $171 \AA$ A, $\mathrm{F}_{\lambda} *$ $\lambda / \mathrm{hc}=2.9 \times 10^{-4}$ photons $\mathrm{cm}^{-2} \mathrm{~s}^{-1}$. With a SEEN survey texp $=35 \mathrm{ksec}$ and $A_{\text {eff }}(171 \AA)=133 \mathrm{~cm}^{2}(\mathrm{G} 20+\mathrm{G} 40$; Figure 8), 1350 source counts are recorded. The background rate is 0.25 counts $\mathrm{cm}^{-2} \mathrm{~s}^{-1} *(0.05 \mathrm{~cm} * 0.1 \mathrm{~cm}$ spot size $) * 2$ grating stripes $=0.00125$ counts $^{-1}$. The $\mathrm{S} / \mathrm{N}=\mathrm{SC} / \sqrt{ }\left(\mathrm{SC}+\mathrm{BG} * \mathrm{t}_{\mathrm{exp}}\right)=1350 / \sqrt{ }(1350+0.00125 * 35000)=35.6$.

\subsection{ESCAPE Surveys}

The ESCAPE baseline data are the SEEN and DEEP spectroscopic surveys (Table 3). Figure 10 demonstrates that ESCAPE achieves a nominal spectral type breakdown of roughly $80 \mathrm{M}$ dwarfs, $40 \mathrm{~K}$ dwarfs, $40 \mathrm{G}$ dwarfs, and $40 \mathrm{~F}$ stars with a range of ages, activity levels and masses. These data provide a direct measure of the absolute EUV stellar flux to better than $40 \%$ after correcting for the ISM (see Appendix). The flare intensity and duration, and coronal dimming lightcurves, are direct observables for stars with sufficient $\mathrm{S} / \mathrm{N}$ and activity. The flux-calibrated spectra and lightcurves are directly input into atmospheric evolution models to calculate expected mass loss rates for different stellar and planetary conditions.

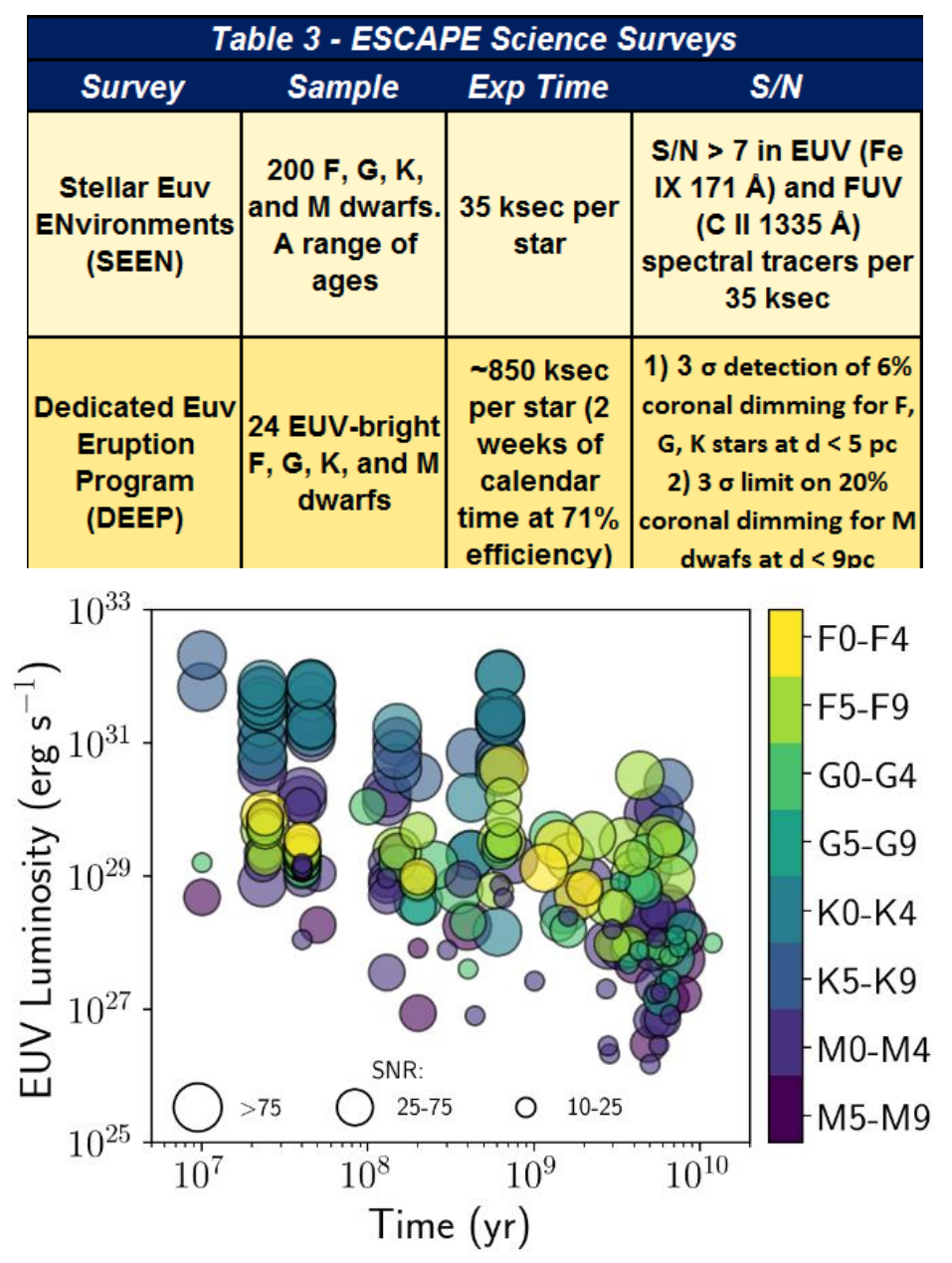

Figure 10 - ESCAPE characterizes the time-variability of the EUV radiation field on stellar evolutionary timescales with the SEEN survey. The EUV luminosity is the estimated $90-360 \AA$ luminosity and the symbol sizes represent the integrated S/N of Fe IX in a $35 \mathrm{ksec}$ SEEN observation. This plot shows our current 200 star target list, with resolution in both age and mass (color-coded), that fulfills the baseline science mission. 


\section{APPENDIX: INTERSTELLAR ABSORPTION WITHIN 100 PARSECS OF THE SUN}

Figure A.1 illustrates the ISM transmission for various neutral hydrogen (H I) column densities. The nearest stars-the $\alpha$ Centauri system - have H I absorbing columns of $10^{17.6} \mathrm{~cm}^{-2}$ (Wood et al. 2005). The solar system resides in the local ISM cloud complex, which itself appears to lie in a lower density "local bubble" (e.g. Bruhweiler \& Cheng, 1988; Redfield \& Linsky, 2000, 2008; Welsh et al., 2010). Despite the physical complexity of the local ISM, integrated H I column density (the most relevant parameter for EUV transmission) rarely exceeds $10^{18.4} \mathrm{~cm}^{-2}$ within $30 \mathrm{pc}$ of the Sun (Wood et al. 2005). Stars within $30 \mathrm{pc}$ encompass almost all habitable zone planets that are candidates for spectroscopic characterization and biomarker detection (LUVOIR Team Interim Report). ESCAPE has the sensitivity to meet the required signal-to-noise and temporal resolution to meet the science objectives outlined in Section 2 for $\sim$ all F, G, K and active M stars out to 30 pc and inactive stars earlier than M5 out to $\sim 12 \mathrm{pc}$. Thus, the interstellar medium is not the limiting factor in our ability to study EUV emission from the most important exoplanet hosts.

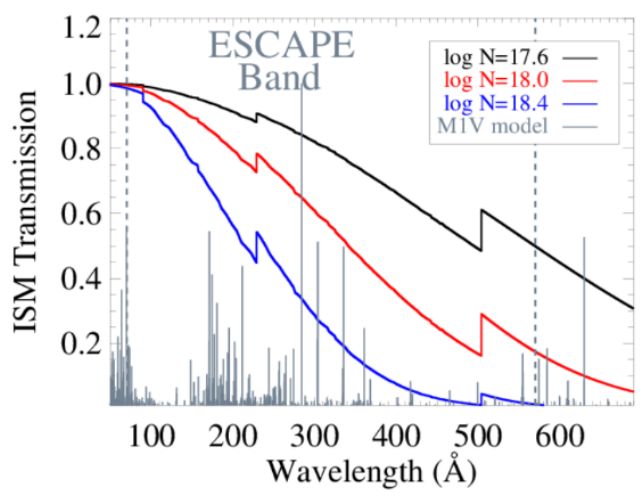

Figure A.1 - Transmission of the local interstellar medium for H I column densities typical of stars inside $30 \mathrm{pc}\left(10^{17.6-18.4} \mathrm{~cm}^{-2}\right.$; Wood et al. 2005). These curves demonstrate that the ISM is more than $20 \%$ transparent to EUV photons over most of the ESCAPE EUV bandpass for most stars inside 30 pc.

Robust corrections for interstellar $\mathrm{H}$ and $\mathrm{He}$ attenuation are critical to computing the true flux of the emergent stellar spectrum. When existing $\mathrm{H}$ column densities are available, uncertainties in the helium abundance and ionization fraction (Dupuis et al. 1995) drive flux uncertainties of $10-20 \%$ at $200 \AA$. Direct Ly $\alpha$ observations from HST-STIS exist for approximately half of our nominal target list, and provide excellent constraints on the neutral hydrogen columns of the ISM. The ESCAPE flux calibration program monitors bright white dwarfs of different temperatures, providing empirical constraints on the $\mathrm{H} / \mathrm{He}$ and helium ionization fractions of the local ISM. We also employ the three-dimensional maps of the local cloud structure developed by Redfield and collaborators for estimation of H I column densities (Redfield \& Linsky 2008). ESCAPE can also observationally constrain ISM attenuation levels with science and calibration spectra. In cool star observations, we compare intensity ratios of stellar emission lines of the same ionization stage and upper energy level (e.g., Fe XIV $210-354 \AA$ A).

We estimate that 'ESCAPE-only' ISM characterization results in absolute flux uncertainties of $\sim 40 \%$, the product of photon noise $(10-15 \%$ for fainter ESCAPE targets, driving the $\mathrm{S} / \mathrm{N}>7$ requirement) and degeneracy between $\mathrm{H} / \mathrm{He}$ abundance and helium ionization fraction $(10-25 \%)$. We emphasize that $40 \%$ absolute uncertainties are a vast advancement over the factor of $3-100$ uncertainty on the EUV irradiance available today.

\section{REFERENCES}

[1] Airapetian, V. S., Glocer, A., Khazanov, G. V., Loyd, R. O. P., France, K., Sojka, J., Danchi,

[2] Aschwanden, M. J., Nitta, N. V., Wuelser, J.-P., Lemen, J. R., Sandman, A., Vourlidas, A., \& Colaninno, R. C. 2009, ApJ, 706, 376

[3] Bourrier, V., de Wit, J., Bolmont, E., Stamenkovi' c, V., Wheatley, P. J., Burgasser, A. J., Delrez, L., Demory, B. O., Ehrenreich, D., \& Gillon, M. 2017, AJ, 154, 121 
[4] Bowyer, S. 1991, ARAA, 29, 59 Bowyer, S., Drake, J. J., \& Vennes, S. 2000, ARAA, 38,

[5] Bowyer, S., Edelstein J., and Lampton, M. 1997, ApJ, 485, 523

[6] Boyd, W. T., Jelinsky, P. N., Finley, D. S., Dupuis, J., Abbott, M., Christian, C., \& Malina, R. F. 1994, SPIE Conference Series, Vol. 2280, ed. O. H. Siegmund \& J. V. Vallerga, 280296

[7] Bruhweiler, F. C. \& Cheng, K.-P. 1988, ApJ, 335, 188

[8] Chassefi`ere, E. 1996, JGR, 101, 26039

[9] Cherenkov, A., Bisikalo, D., Fossati, L., \& Mo“stl, C. 2017, ApJ, 846, 31

[10] Crosley, M. K. \& Osten, R. A. 2018, ApJ, 856, 39

[11] Dissauer, K., Veronig, A. M., Temmer, M., Podladchikova, T., \& Vanninathan, K. 2018a, ApJ, 855, 137

[12] Dissauer, K., Veronig, A. M., Temmer, M., Podladchikova, T., \& Vanninathan, K. 2018b, ApJ, 863, 169

[13] Drake, J. J., Kashyap, V. L., Wargelin, B. J., \& Wolk, S. J. 2019, arXiv e-prints, arXiv: 1901.05525

[14] Dupuis, J., Vennes, S., Bowyer, S., Pradhan, A. K., \& Thejll, P. 1995, ApJ, 455, 574

[15] Ertley, C., Siegmund, O. H. W., Schwarz, J., Mane, A. U., Minot, M. J., O’Mahony, A., Craven, C. A., \& Popecki, M. 2015, SPIE Conference Series, Vol. 9601, 96010S

[16] Fontenla, J. M., Linsky, J. L., Witbrod, J., France, K., Buccino, A., Mauas, P., Vieytes, M., \& Walkowicz, L. M. 2016, ApJ, 830, 154

[17] France, K., Arulanantham, N., Fossati, L., Lanza, A. F., Loyd, R. O. P., Redfield, S., \& Schneider, P. C. 2018, ApJS, 239, 16

[18] France, K., Froning, C. S., Linsky, J. L., Roberge, A., Stocke, J. T., Tian, F., Bushinsky, R., D'esert, J.-M., Mauas, P., Vieytes, M., \& Walkowicz, L. M. 2013, ApJ, 763, 149

[19] France, K., Parke Loyd, R. O., Youngblood, A., Brown, A., Schneider, P. C., Hawley, S. L., Froning, C. S., Linsky, J. L., Roberge, A., Buccino, A. P., Davenport, J. R. A., Fontenla, J. M., Kaltenegger, L., Kowalski, A. F., Mauas, P. J. D., Miguel, Y., Redfield, S., Rugheimer, S., Tian, F., Vieytes, M. C., Walkowicz, L. M., \& Weisenburger, K. L. 2016, ApJ, 820, 89

[20] Garcia-Sage, K., Glocer, A., Drake, J. J., Gronoff, G., \& Cohen, O. 2017, ApJL, 844, L13

[21] Green, J. C., Froning, C. S., Osterman, S., Ebbets, D., Heap, S. H., Leitherer, C., Linsky, J. L., Savage, B. D., Sembach, K., Shull, J. M., Siegmund, O. H. W., Snow, T. P., Spencer, J., Stern, S. A., Stocke, J., Welsh, B., B' eland, S., Burgh, E. B., Danforth, C., France, K., Keeney, B., McPhate, J., Penton, S. V., Andrews, J., Brownsberger, K., Morse, J., \& Wilkinson, E. 2012, ApJ, 744, 60

[22] Haisch, B. M., Linsky, J. L., Bornmann, P. L., Stencel, R. E., Antiochos, S. K., Golub, L., \& Vaiana, G. S. 1983, ApJ, 267, 280

[23] Harman, C. E., Schwieterman, E. W., Schottelkotte, J. C., \& Kasting, J. F. 2015, ApJ, 812, 137

[24] Harra, L. K., Schrijver, C. J., Janvier, M., Toriumi, S., Hudson, H., Matthews, S., Woods, M. M., Hara, H., Guedel, M., \& Kowalski, A. 2016, SolPhys, 291, 1761

[25] Hettrick, M. \& Bowyer, S. 1984, in NASA Conference Publication, ed. J. M. Mead, R. D. Chapman, \& Y. Kondo, Vol. 2349, 529

[26] Jensen, P. L., Ellwood, J. M., \& Peacock, A. 1989, SPIE Conference Series, Vol. 1160, ed. R. B. Hoover, 525-0

[27] Khodachenko, M. L., Ribas, I., Lammer, H., Grießmeier, J.-M., Leitner, M., Selsis, F., Eiroa, 
C., Hanslmeier, A., Biernat, H. K., \& Farrugia, C. J. 2007, Astrobiology, 7, 167

[28] Kobayashi, K., Winebarger, A. R., Savage, S., Champey, P., Cheimets, P. N., Hertz, E., Bruccoleri, A. R., Scholvin, J., Golub, L., \& Ramsey, B. 2018, SPIE Conference Series, Vol. 10699, 1069927

[29] Koglin, J. E., An, H., Blaedel, K. L., Brejnholt, N. F., Christensen, F. E., Craig, W. W., Decker, T. A., Hailey, C. J., Hale, L. C., \& Harrison, F. A. 2009, SPIE Conference Series, Vol. 7437, 74370C

[30] Lammer, H., Bredeh oft, J. H., Coustenis, A., Khodachenko, M. L., Kaltenegger, L., Grasset, O., Prieur, D., Raulin, F., Ehrenfreund, P., \& Yamauchi, M. 2009, aapr, 17, 181

[31] Lammer, H., Lichtenegger, H. I. M., Kulikov, Y. N., Grießmeier, J.-M., Terada, N., Erkaev, N. V., Biernat, H. K., Khodachenko, M. L., Ribas, I., Penz, T., \& Selsis, F. 2007, Astrobiology, 7, 185

[32] Lammer, H., Zerkle, A. L., Gebauer, S., Tosi, N., Noack, L., Scherf, M., Pilat-Lohinger, E., Güdel, M., Grenfell, J. L., \& Godolt, M. 2018, aapr, 26, 2

[33] Linsky, J. L., Fontenla, J., \& France, K. 2014, ApJ, 780, 61

[34] Loyd, R. O. P., France, K., Youngblood, A., Schneider, C., Brown, A., Hu, R., Segura, A., Linsky, J., Redfield, S., \& Tian, F. 2018a, ApJ, 867, 71

[35] Loyd, R. O. P., Shkolnik, E. L., Schneider, A. C., Barman, T. S., Meadows, V. S., Pagano, I., \& Peacock, S. 2018b, ApJ, 867, 70

[36] Mason, J. P., Woods, T. N., Chamberlin, P. C., Jones, A., Kohnert, R., Schwab, B., Sewell, R., Caspi, A., Moore, C. S., Palo, S., Solomon, S. C., \& Warren, H. 2019, arXiv e-prints, arXiv: 1905.01345

[37] Mason, J. P., et al., 2019b, in preparation

[38] McCandliss, S. R., Feldman, P. D., Weaver, H., Fleming, B., Redwine, K., Li, M. J., Kutyrev, A., \& Moseley, S. H. 2016, AJ, 152, 65

[39] McEntaffer, R. L. 2019, Journal of Astronomical Telescopes, Instruments, and Systems, 5, 1

[40] Meadows, V. S., Arney, G. N., Schwieterman, E. W., Lustig-Yaeger, J., Lincowski, A. P., Robinson, T., Domagal-Goldman, S. D., Deitrick, R., Barnes, R. K., \& Fleming, D. P. 2018, Astrobiology, 18, 133

[41] Miles, D. M., McCoy, J. A., McEntaffer, R. L., Eichfeld, C. M., Lavallee, G., Labella, M., Drawl, W., Liu, B., DeRoo, C. T., \& Steiner, T. 2018, ApJ, 869, 95

[42] Morrison, R. \& McCammon, D. 1983, ApJ, 270, 119

[43] Moschou, S.-P., Drake, J. J., Cohen, O., Alvarado-Gomez, J. D., \& Garraffo, C. 2017, ApJ, 850,191

[44] Odert, P., Leitzinger, M., Hanslmeier, A., \& Lammer, H. 2017, MNRAS, 472, 876

[45] Osten, R. A. \& Wolk, S. J. 2015, ApJ, 809, 79

[46] Redfield, S. \& Linsky, J. L. 2000, ApJ, 534, 825

[47] Redfield, S. \& Linsky, J. L. 2008, ApJ, 673, 283

[48] Reinard, A. A. \& Biesecker, D. A. 2009, ApJ, 705, 914

[49] Ribas, I., Gregg, M. D., Boyajian, T. S., \& Bolmont, E. 2017, A\&A, 603, A58

[50] Sanz-Forcada, J., Brickhouse, N. S., \& Dupree, A. K. 2003, ApJS, 145, 147

[51] Sanz-Forcada, J., Micela, G., Ribas, I., Pollock, A. M. T., Eiroa, C., Velasco, A., Solano, E., \& Garc' 1a-A lvarez, D. 2011, A\&A, 532, A6

[52] Segura, A., Kasting, J. F., Meadows, V., Cohen, M., Scalo, J., Crisp, D., Butler, R. A. H., \& Tinetti, G. 2005, Astrobiology, 5, 706 
[53] Segura, A., Walkowicz, L. M., Meadows, V., Kasting, J., \& Hawley, S. 2010, Astrobiology, 10,751

[54] Siegmund, O. H., Vallerga, J. V., Everman, D. E., \& Hull, J. 1989, in Soci- ety of PhotoOptical Instrumentation Engineers (SPIE) Conference Series, Vol. 1140, Society of PhotoOptical Instrumentation Engineers (SPIE) Conference Series, ed. R. Be- nattar, 106-0

[55] Siegmund, O. H. W., Ertley, C., Vallerga, J. V., Schindhelm, E. R., Harwit, A., Fleming, B. T., France, K. C., Green, J. C., McCandliss, S. R., \& Harris, W. M. 2017, SPIE Conference Series, Vol. 10397, 1039711

[56] Siegmund, O. H. W., McPhate, J. B., Tremsin, A. S., Vallerga, J. V., Ertley, C. D., Richner, N. J., Gerard, T. M., Frisch, H. J., Elam, J. W., \& Mane, A. U. 2015, Nuclear Instruments and Methods in Physics Research A, 787, 110

[57] Siegmund, O. H. W., Vallerga, J., \& Wargelin, B. 1988, IEEE Transactions on Nuclear Science, 35,524

[58] Sirk, M. M., Vallerga, J. V., Finley, D. S., Jelinsky, P., \& Malina, R. F. 1997, ApJS, 110, 347

[59] Tian, F., France, K., Linsky, J. L., Mauas, P. J. D., \& Vieytes, M. C. 2014, Earth and Planetary Science Letters, 385, 22

[60] Tilley, M. T. 2018, PhD thesis, University of Washington

[61] Vallerga, J. V., McPhate, J. B., Martin, A. P., Gaines, G. A., Siegmund, O. H., Wilkinson, E., Penton, S., \& Beland, S. 2001, SPIE Conference Series, Vol. 4498, ed. O. H. Siegmund, S. Fineschi, \& M. A. Gummin, 141-151

[62] Vidal-Madjar, A., D' esert, J. M., Lecavelier des Etangs, A., H'ebrard, G., Ballester, G. E., Ehrenreich, D., Ferlet, R., McConnell, J. C., Mayor, M., \& Parkinson, C. D. 2004, ApJL, 604, L69

[63] Wilkinson, E., Green, J. C., \& Cash, W. 1993, ApJS, 89, 21110

[64] Wood, B. E., Redfield, S., Linsky, J. L., Mu 1ler, H.-R., \& Zank, G. P. 2005, ApJS, 159, 118

[65] Woods, T. N., Chamberlin, P. C., Harder, J. W., Hock, R. A., Snow, M., Eparvier, F. G., Fontenla, J., McClintock, W. E., \& Richard, E. C. 2009, grl, 36, L01101

[66] Woods, T. N., Eparvier, F. G., Hock, R., Jones, A. R., Woodraska, D., Judge, D., Didkovsky, L., Lean, J., Mariska, J., \& Warren, H. 2012, SolPhys, 275, 115

[67] Woods, T. N., Hock, R., Eparvier, F., Jones, A. R., Chamberlin, P. C., Klimchuk, J. A., Didkovsky, L., Judge, D., Mariska, J., \& Warren, H. 2011, ApJ, 739, 59 